\title{
Provision of dental hospital services during the COVID-19 epidemic
}

\section{Albert Yeung}

\section{A commentary on}

\section{Yang Y, Zhou Y, Liu X, Tan J.}

Health services provision of 48 public tertiary dental hospitals during the COVID-19 epidemic in China. Clin Oral Investig 2020; 24:

1861-1864.

\begin{abstract}
Data sources Information about health services provision of public tertiary dental hospitals in China mainland was retrieved through a web search engine.

Data analysis The status of non-emergency dental services, emergency dental services and online professional consultation was recorded and analysed. If telehealth was provided, the charge, means of access, service time and service content were investigated. The geographical distribution of hospitals was recorded and classified. Results A total of 48 dental hospitals were included in the study. All 48 dental hospitals suspended general non-emergency dental treatment. They provided emergency dental services only.

Conclusions There was a significant change in the health service provision of Chinese dental hospitals during the COVID-19 epidemic and wider use of telehealth in the eastern region.
\end{abstract}

\section{Commentary}

The World Health Organisation declared the coronavirus disease (COVID-19) outbreak as a public health emergency of international concern on 30 January 2020 and a global pandemic on 11 March 2020. This has led to a reduction in dental services all over the world.

At present, there are four main dental service providers in China: dental hospitals, dental departments in general hospitals, chain dental clinics and individual dental clinics. At the end of 2017, there were 689 dental hospitals of which 164 were public dental hospitals. ${ }^{1}$

This study investigated 48 public tertiary dental hospitals in China through a web search engine. The sample size appears to be small as the total number of public dental hospitals in China should be over $100 .{ }^{1}$ The authors did not provide a clear definition of public tertiary dental hospitals.

Teledentistry can be defined as the remote provision of dental care, advice, or treatment through the medium of information technology, rather than through direct personal contact with any patient(s) involved. ${ }^{2}$ It can be provided in a number of ways, such as live video (synchronous), store-and-forward (asynchronous), remote patient monitoring (RPM) or mobile health (mHealth). ${ }^{3}$
Practice points

- Teledentistry can assist in remote assessment (triage) and continuity of dental care during the time of COVID-19 physical distancing.

- If a dental visit is needed, teledentistry would allow all the paperwork, signatures, explanations, and consent to be handled before the visit. This would lower the risk of transmission for both patients and oral healthcare providers.

It has the potential to eliminate the disparities in oral healthcare between rural and urban communities.

The authors of this paper reported that $69 \%$ of public tertiary dental hospitals offered free online professional consultations during the COVID-19 epidemic. The penetration rate was significantly higher in the eastern region (87\%) than that of central and western regions (52\%).

For patients with COVID-19, or those who suspect they may be infected, teledentistry can assist in remote assessment and continuity of care. For those not infected with COVID-19, particularly those at higher risk of being affected (for example, patients with pre-existing medical conditions), teledentistry can provide rapid access to a safe course of action in congested hospital or dental practice waiting rooms. Effective teledentistry requires a good infrastructure on both sides of the consultation. ${ }^{4}$

Teledentistry can supplement traditional methods of oral diagnosis and referral for patients who might not have access to care. The challenge is how to use this technology to add to what is already provided, by incorporating alternative service delivery systems, to address demands for oral healthcare for underserved populations using non-traditional settings, and transcending social, geographic and cultural barriers. Potentially this could mean reduced costs, improved patient outcomes and greater access to quality oral healthcare. ${ }^{5}$

\section{Author affiliation \\ ${ }^{1}$ Department of Public Health, NHS Lanarkshire, Kirklands, Fallside Road, Bothwell G71 8BB, UK \\ Email: albert.yeung@lanarkshire.scot.nhs.uk}

\section{References}

1. Huajing Information Network. Analysis of the supply and demand situation of China's oral health care market in 2018, private dental hospitals will develop rapidly. 2019. Available at https://m.huaon.com/detail/411338.html (accessed May 2020).

2. Khan S A, Omar H. Teledentistry in Practice: Literature Review. Telemed J E Health 2013; 19: 565-567.

3. American Dental Association. ADA Policy on Teledentistry. Available at https://www. ada.org/en/about-the-ada/ada-positions-policies-and-statements/statement-onteledentistry (accessed May 2020).

4. Maret $D$, Peters $O A$, Vaysse $F$, Vigarios $E$. Integration of telemedicine into the public health response to COVID-19 must include dentists. Int Endod / 2020; 53: 880-881.

5. Mariño R. e-(Oral) Health: A World-Wide Overview [Guest Editorial]. J Int Soc Telemed eHealth 2019; 7: e7. 